NASZA DERMATOLOGIA Online OUR DERMATOLOGY Online

Source of Support: Nil

Competing Interests: None

\section{SCORING SYSTEMS IN BULLOUS DERMATOSES}

\author{
Sunderamoorthy Srinivasan, Ramamoorthy Mathumathy
}

Chettinad Hospital \& Research Institute, Kelambakkam, Chennai, India

Corresponding authors: Prof. Sunderamoorthy Srinivasan Dr. R. Mathumathy hamsrini@yahoo.co.in drmathumathy@yahoo.co.in
Sir

These group of disorders are chronic blistering auto immune disorders characterized histologically by intra epidermal/ sub epidermal blister formation. They have circulatory auto antibodies against distinct adhesion molecules of epidermis and BMZ. They are potentially life threatening, characterized by widespread blisters and erosions of skin and mucosa.

Pemphigus being clinically heterogenous group of disorder, comprehensive scoring systems are needed to assess the severity of disease for effective monitoring of treatment.

Pemphigus Disease Area Index (Tabl. I) integrates cutaneous with mucosal disease in well-defined anatomical locations, assesses number and sizes of lesions and also scores postinflammatory hyperpigmentation of resolving lesions.

Numerous scoring systems (Tabl. II-IV) have been described in literature at different times to score disease activity of pemphigus.

\section{SCORING SYSTEMS}

\section{ABSIS}

(Auto immune bullous skin disorder intensity score)

\section{SKIN INVOLVEMENT}

2 parameters

* The extent of affected area (calculated by rule of nine)

* Quality of skin lesions

$\%$ of BSA X weighing factor

Weighing factor

1.5- for erosive,exudative lesions, blisters, positive Nikolsky sign.

1 - for erosive, dry lesions

0.5 - re epithelialized lesions

$0-150$ points

Compare scores from initial scores with treatment to monitor disease activity.

\section{ORAL INVOLVEMENT}

1st Mucosal score-extent of lesions (0-11)

1- upper gingival mucosa

2-lower gingival mucosa

3-upper lip mucosa

4-lower lip mucosa

5-left buccal mucosa

6-right buccal mucosa

7-tongue

8 -floor of mouth

9-hard palate

10-soft palate

11-pharynx

Rating

0 -no lesion

1-presence of lesion

2nd Mucosal involvement- severity of lesion (0-45)

1 point-if pain/bleeding always occurred

0.5 point-pain/bleeding occurred sometimes

0 point-no pain

Types of food -

Water $-1 * x$

Soup $-2 * x$

Yoghurt $-3 * x$

Custard $-4 * x$

Smashed potatoes/scrambled egg $-5^{*} \mathrm{x}$

Baked fish $-6^{*} x$

White bread $-7 * \mathrm{x}$

Apple/raw carrot $-8 * \mathrm{x}$

Whole grain bread $-9 * x$ 


\begin{tabular}{|c|c|c|c|}
\hline Skin & Activity & & Damage \\
\hline \multicolumn{4}{|l|}{ PDAI-skin } \\
\hline \multirow[t]{2}{*}{$\begin{array}{l}\text { Anatomical } \\
\text { location }\end{array}$} & Erosion/blister or new erythema & \multirow{3}{*}{ Number of lesions, if $<3$} & $\begin{array}{l}\text { Post-inflamatory hyperpigmentation or erythema from } \\
\text { resolving lesions }\end{array}$ \\
\hline & $\begin{array}{l}0=\text { Absent } \\
1=1-3 \text { lesions, up to one } ? 2 \mathrm{~cm} \text { in any diameter, } \\
\text { none }>6 \mathrm{~cm} \\
2=2-3 \text { lesions at laest two }>2 \mathrm{~cm}, \text { none }>6 \mathrm{~cm} \\
3=>3 \text { lesions, none }>6 \mathrm{~cm} \\
5=>3 \text { lesions, and } / \text { or at least one }>6 \mathrm{~cm} \\
10=>3 \text { lesions, and } / \text { or at least one }>16 \mathrm{~cm} \text { or } \\
\text { entire area }\end{array}$ & & $\begin{array}{l}0=\text { Absent } \\
1=\text { Present }\end{array}$ \\
\hline $\begin{array}{l}\text { Ears } \\
\text { Nose } \\
\text { Rest of face } \\
\text { Neck } \\
\text { Chest } \\
\text { Andomen } \\
\text { Back/buttocks } \\
\text { Arms } \\
\text { Hands } \\
\text { Legs } \\
\text { Feet } \\
\text { Genitals } \\
\text { Total skin }\end{array}$ & $/ 120$ & & $/ 12$ \\
\hline \multicolumn{4}{|l|}{ PDAI-skin } \\
\hline Scalp & Erosion/blister or new erythema & \multirow[t]{3}{*}{ Number of lesions, if $<3$} & $\begin{array}{l}\text { Post-inflamatory hyperpigmentation or erythema from } \\
\text { resolving lesions }\end{array}$ \\
\hline \multirow[b]{2}{*}{ Total scalp } & $\begin{array}{l}0=\text { Absent } \\
1=\text { In one quadrat } \\
2=\text { Two quadrats } \\
3=\text { Three quadrats } \\
4=\text { Whole skull } \\
10=\text { At least one lesion } ? 6 \mathrm{~cm}\end{array}$ & & $\begin{array}{l}0=\text { Absent } \\
1=\text { Present }\end{array}$ \\
\hline & $/ 10$ & & $/ 1$ \\
\hline \multicolumn{4}{|c|}{ PDAI-mucous membranes } \\
\hline $\begin{array}{l}\text { Anatomical } \\
\text { location }\end{array}$ & $\begin{array}{l}\text { Erosion/blister } \\
0=\text { Absent } \\
1=1 \text { lesion } \\
2=2-3 \text { lesions } \\
5=>3 \text { lesions or } 2 \text { lesions }>2 \mathrm{~cm} \\
10=\text { entire area }\end{array}$ & \multirow[t]{2}{*}{ Number of lesions, if $<3$} & \\
\hline $\begin{array}{l}\text { Eyes } \\
\text { Nose } \\
\text { Buccal mucosa } \\
\text { Hard palate } \\
\text { Soft palate } \\
\text { Upper gingiva } \\
\text { Lower gingiva } \\
\text { Tongue } \\
\text { Floor of mouth } \\
\text { Labial mucosa } \\
\text { Posterior pharynx } \\
\text { Anogenital } \\
\text { Total mucosa }\end{array}$ & $/ 120$ & & \\
\hline
\end{tabular}




\begin{tabular}{|c|c|c|}
\hline \multicolumn{2}{|c|}{ Severity } & \multirow[t]{2}{*}{ Antibody Titer Level } \\
\hline Oral & Skin & \\
\hline $0=\mathrm{Nil}$ & $0=\mathrm{Nil}$ & \multirow[t]{4}{*}{ Dsg1 and Dsg3 levels as assessed by ELISA } \\
\hline $1=<3$ Erosions & $1=<5$ Erosions & \\
\hline $2=3-4$ Erosions & $2=5-20$ Erosions & \\
\hline $3=>10$ Erosions & $3=>20$ Erosions & \\
\hline
\end{tabular}

Oral score

0 - No mucosal involvement

1 - Minimal disease (only bucal mucosa, labiogingival, lingual, palatal, pharyngeal)

2 - Moderate disease (bucal and labiogingival, lingual, palatal or pharyngeal)

3 - Severe disease (extensive oral erosions, i.e., $>3$ mucosal sites affected)

\begin{tabular}{l}
\hline Skin score \\
\hline 0 - Quescent disease \\
1 - Minimal disease $(<10 \%$ BSA $)$ \\
2 - Moderate disease $(11-30 \%$ BSA) \\
3 - Severe disease $(>30 \%$ BSA $)$
\end{tabular}

Table III. Kumar's Scoring System.

\begin{tabular}{|l|l|l|}
\hline \multicolumn{1}{|c|}{ Severity } & \multicolumn{1}{|c|}{ Cutaneus involvement } & \multicolumn{1}{c|}{ Mucosal involvement } \\
\hline Mild (1+) & $\begin{array}{l}10 \% \text { BSA, able to carry daily routine without discomfort } \\
\text { or localisation to oral mucosa only }\end{array}$ & $\begin{array}{l}\text { Only localised to buccal mucosa, no difficulty in swallowing or } \\
\text { chewing }\end{array}$ \\
\hline Moderate (2+) & $\begin{array}{l}10-25 \% \text { BSA along with oral mucosal involvement, able } \\
\text { to carry out daily routine with discomfort }\end{array}$ & $\begin{array}{l}\text { Buccal and gingivolabial mucosal involvement, difficulty in solid } \\
\text { food intake }\end{array}$ \\
\hline Severe (3+) & $\begin{array}{l}\text { 25-50\% BSA along with oral mucosal involvement, } \\
\text { unable to carry out daily routine }\end{array}$ & $\begin{array}{l}\text { Extensive oral mucosal involvement, difficulty in semisolid food } \\
\text { inake }\end{array}$ \\
\hline Extensive (4+) & $\begin{array}{l}\text { 50\% BSA along with mucosal involvement, bedridden or } \\
\text { has complications }\end{array}$ & $\begin{array}{l}\text { Extensive oral mucosal involvement, other mucosal involvement, } \\
\text { difficulty in swallowing liquids also (unable to take anything } \\
\text { orally) }\end{array}$ \\
\hline
\end{tabular}

Copyright by Sunderamoorthy Srinivasan, et al. This is an open access article distributed under the terms of the Creative Commons Attribution License, which permits unrestricted use, distribution, and reproduction in any medium, provided the original author and source are credited. 